\title{
A Parametric Programming Technique For Efficient CNC Machining Operations
}

\author{
Manocher Djassemi \\ Department of Industrial and Engineering Technology \\ Murray State University \\ P.O.Box 9 \\ Murray, KY 42071
}

\begin{abstract}
In this paper the application of parametric programming to $\mathrm{CNC}$ machining is discussed. As one of the less frequently utilized features of CNC machines, parametric programming has the potential to increase the efficiency of CNC operations. This feature is particularly beneficial to companies with group technology manufacturing where parts with similar design or operational requirements are processed within a machine cell. Using two case studies, the capabilities of parametric programming for CNC machines are illustrated.
\end{abstract}

Key Words: Parametric Programming, CNC machining, Group Technology

\section{INTRODUCTION}

Parametric programming, as a feature of modern Computer Numerical Control (CNC) machines, has the potential to bring higher efficiency to manufacturing industries. The application of parametric programming to $\mathrm{CNC}$ operations is possible in several ways. These include generating a single CNC program for parts with similar design, inventing macros for machining custom design features, and developing subprograms for a group of parts that are not similar in design but require similar machining operations. In all these applications, parametric programming can significantly reduce the part programming time and effort which in turn leads to shorter throughput and product development times. These applications particularly fit group technology manufacturing in which similar parts are grouped into part families and then processed by a number of machine tools within a cell or by a single multi-tasking machining center. The two common approaches in group technology are grouping based on design similarity, and grouping based on similar machining requirements. Parametric programming can be applied to part families formed by either of the two grouping approaches, as illustrated in this study.

\section{WHAT IS PARAMETRIC PROGRAMMING?}

Parametric programming is a $\mathrm{G} / \mathrm{M}$ code programming in which axis position $(x, y, z, a$, etc.), feed and speed functions can be specified by a parametric expression. Similar to computer programming languages such as Pascal or $\mathbf{C}$, computer-related features such as variables, arithmetic, logic statements and looping are available in parametric programming (Lynch, 1995).

Most CNC machines provide a parametric programming feature that allows the user to load a part program or a subprogram to the machine controller. The part program is then called up whenever a similar part is machined or similar operation has to be performed on one or more parts. The process involves a simple entry of parameter values into the machine controller. For example, several parts may require machining a pocket with different sizes or drilling a hole circle pattern of various diameters and number of holes. A single parametric subprogram can be called up from the main program to machine these part features. Upon loading the main program, the values of parameters associated with a feature such as 
length, width and depth of a pocket are entered; then, these values are automatically transferred from the main program to the parametric subprogram. This approach eliminates the redundant codes in part program and reduces the size of the program and programming time.

In this study, two groups of parts will be used to illustrate the benefits of parametric programming for manufacturing plants with CNC machines and machining or turning centers.

\section{APPLICATION OF PARAMETRIC PROGRAMMIING}

\section{Feature-Based CNC Programming}

Parametric programming technique allows the creation of program modules for specific part features such as a hole, slots, pockets, threads, etc. (Fig. 1). These pre-programmed machining modules can be selected based on design features. Each time a new part is designed, the CNC program calls parametric modules associated with design features. This approach eliminates the need for programming features already available in parametric modules.

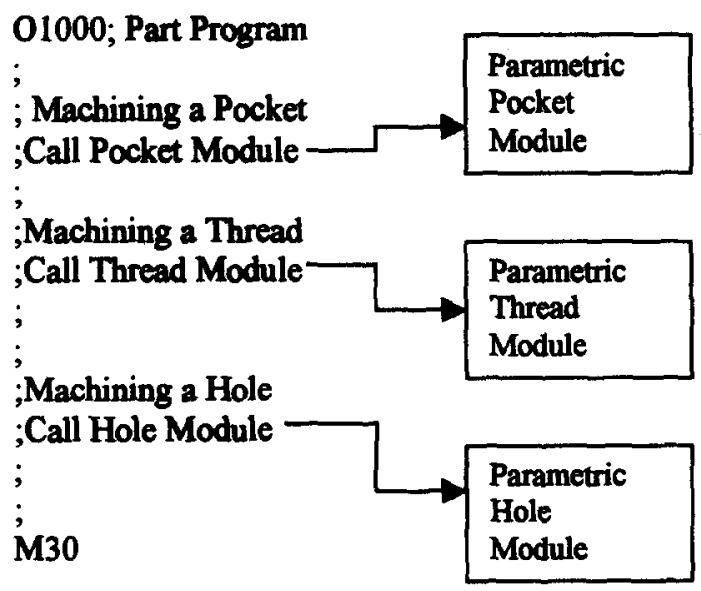

Fig.1 Feature-Based CNC Program Using Parametric Modules

\section{Repeated Features}

When a design feature is repeated among a number of products, the use of parametric programming is a major productivity advantage over conventional $\mathbf{G} / \mathbf{M}$ code programming where tool position coordinates would have to be changed every time a design engineer changes the part drawing. With the parametric programming approach, similar parts can be machined using a single program. This eliminates redundant programming and reduces the number of duplicate programs, which can be significant in companies producing a large variety of parts.

\section{CASE STUDIES}

To illustrate the application of parametric programming to CNC machining two cases are presented below.

\section{Case 1}

A group of non-similar parts are depicted in Fig. 2. These parts require different machining operations except for machining a common center hole . Figure 3 shows a CNC part program for machining a hole using a conventional $\mathbf{G} / \mathbf{M}$ code programming approach. This program must be modified for each part. Figure 4 shows the same program in parametric format (Miltronics, 1990). The difference between the two formats is that the parametric program can be called from any program to machine a hole of any size at any location, while the conventional program can only machine a specific hole size. The advantage for CNC machine users is that the operator will only enter

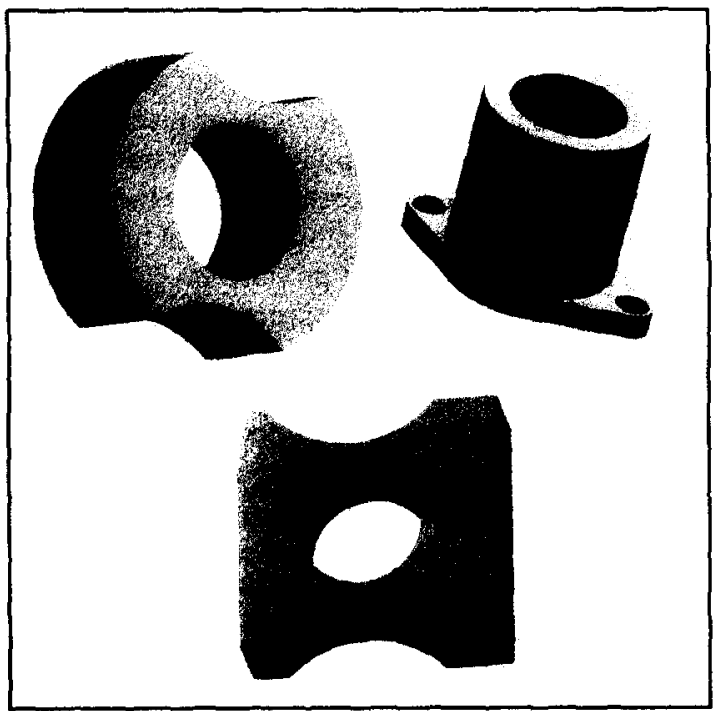

Fig. 2. Parts with similar design feature 
the value of hole diameter, depth of hole, feedrate and spindle speed (parameters P10 to P16). Tool position coordinates in the part program would not be changed as it is the case in conventional G/M programming approach.

\section{0 \\ G92 X0 Y0 Z1 \\ $\mathrm{S} 1000 \mathrm{MO3}$ \\ G00 X2.5 Y1 \\ M06 T01 \\ G43 H1 Z.1 \\ G01 Z-.5 F10 \\ Y2.125 F15 \\ G02 J-2.125 \\ G01 Y1.0 \\ M30}

Fig. 3. Conventional G/M program for machining a hole

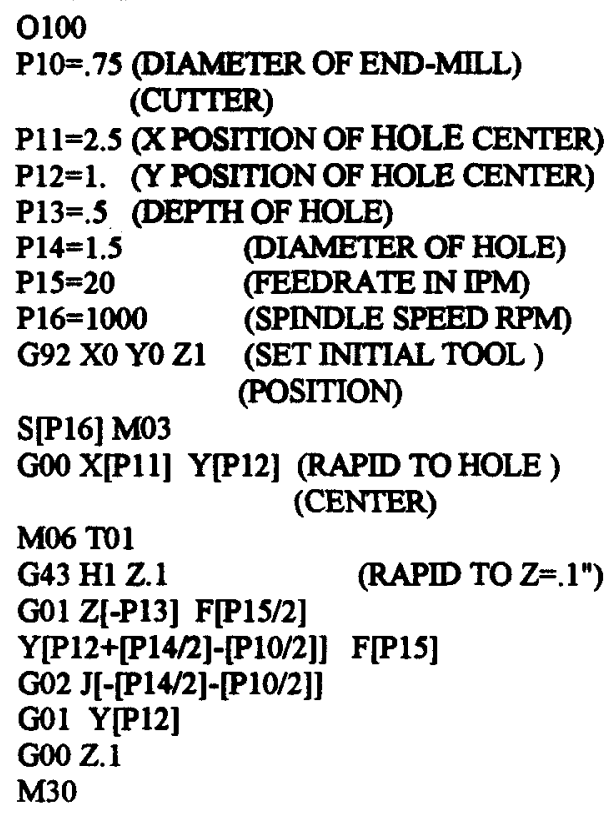

Fig. 4. Parametric program for machining a hole

\section{Case 2}

A group of similar parts are shown in Fig. 5. This group represents a family of parts with similar design features such as thread and side steps. By changing the value of major parameters, such as diameter and overall length, a larger number of parts can be included within the same part family. The average number of NC instructions for machining all parts is 82 lines (Fig.6), while the number of NC instruction in corresponding parametric program is 38 lines (Fig.7).

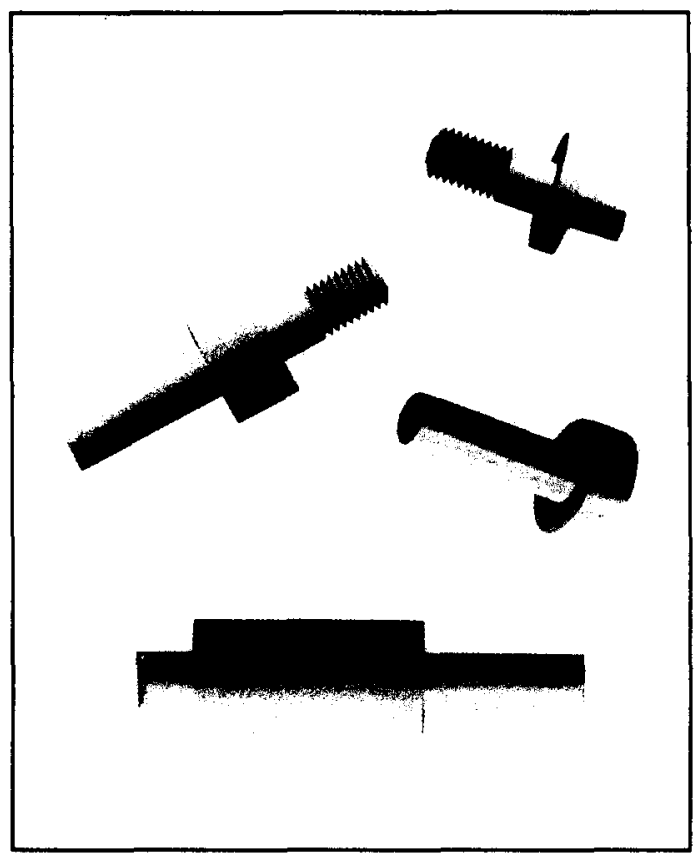

Fig. 5 Parts with similar design

\section{(Cylindrical Parts)}

NO02 GSO X14. Z5. S1000

N003 G00 T0505 (Left Shoulder)

N004 G97 S1000 M03

N005 G00 X1.4 Z-.1

N006 G96 $\$ 1000$

N007 G99

N008 G71 P008 Q030 U0. W0. D2000 F.01

N008 G00 X1.044

N009 G99 G01 X.644 F.003

.........

(.......

-

$\ldots \ldots \ldots$

N128 G00 X1.5 (Cutoff the Part)

N129 Z-2.5

N130 G01 X0 F.005

N131 G00 G40 X10 Z10 T1300

N132 M05

N133 M30

Fig. 6. Partial part program for machining family of parts in Fig. 5 


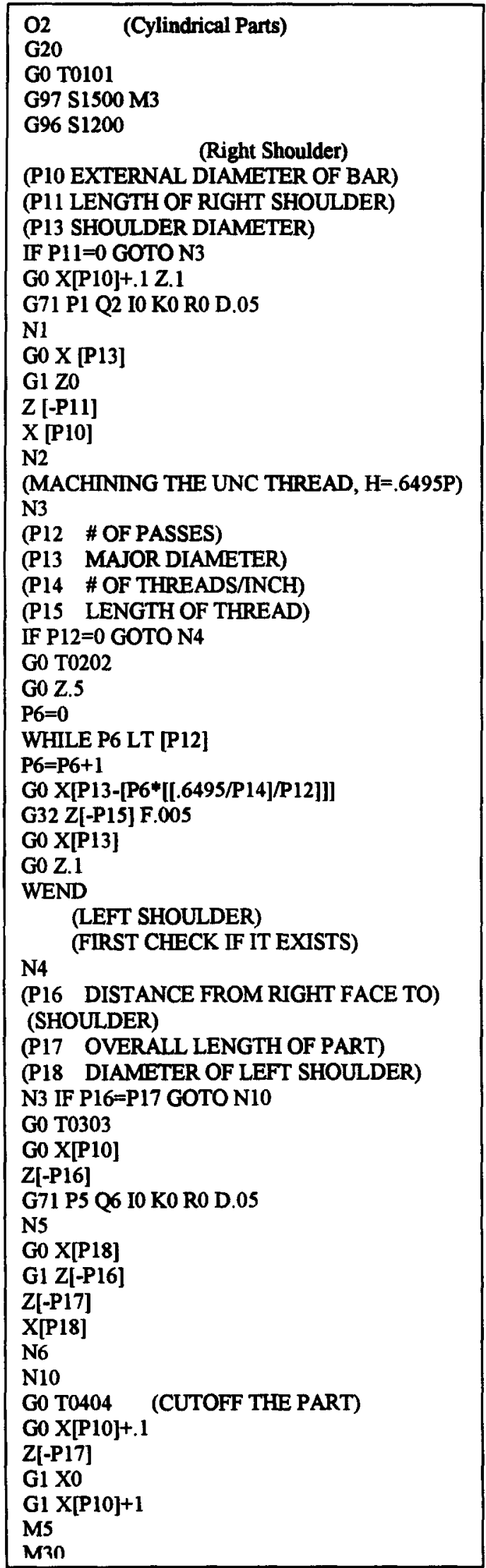

Fig. 7. Parametric program for parts in Fig. 4

\section{CONCLUSION}

In today's competitive economy, manufacturing companies have no alternative other than taking advantage of new technologies in improving the efficiency of their operations. Parametric programming is one of the less utilized features of modern CNC machines and can serve as a tool to improve the efficiency of programming $\mathrm{CNC}$ machines. This paper shows the characteristics of parametric programming. Two cases of the application of parametric programming are used to illustrates the differences between a conventional $\mathrm{CNC}$ program and its equivalent parametric program, and to show the benefits of this programming technique in terms of using a single program for machining a number of parts, shorter programs, and lower programming time. Case studies from successful applications of parametric programming in industry would be appropriate to further publicize this technology among $\mathrm{CNC}$ machine users.

\section{REFERENCES:}

Lynch, Mike (1995). Managing computer control operations, how to get the most out of your CNC machine tools. Society of Manufacturing Engineers, Dearborn, MI. Miltronics Manufacturing Company (1990). Centurion V operation manual, version 1.3. Chanhassen, $\mathrm{MN}$. 